\title{
Increased IncRNA HOTAIR expression promotes the chemoresistance of multiple myeloma to dexamethasone by regulating cell viability and apoptosis by mediating the JAK2/STAT3 signaling pathway
}

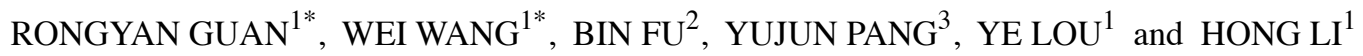 \\ ${ }^{1}$ Department of Hematology, Daqing Oilfield General Hospital, Daqing, \\ Heilongjiang 163001; ${ }^{2}$ Department of Hematology, Heze Municipal Hospital, Heze, Shandong 274000; \\ ${ }^{3}$ Department of Central Laboratory, Daqing Oilfield General Hospital, Daqing, Heilongiiang 163001, P.R. China
}

Received December 12, 2018; Accepted May 2, 2019

DOI: $10.3892 / \mathrm{mmr} .2019 .10603$

\begin{abstract}
Multiple studies have shown that HOX antisense intergenic RNA (HOTAIR), an oncogenic long non-coding RNA (lncRNA), is dysregulated in leukemia and is involved in tumor progression. The aim of the present study was to determine whether HOTAIR could serve as a novel biomarker for the diagnosis of multiple myeloma (MM), and to investigate its role in regards to MM cell viability and chemoresistance to dexamethasone (DEX). The results revealed that the expression of HOTAIR was significantly upregulated in serum, bone marrow and primary $\mathrm{CD} 138^{+}$cells from $\mathrm{MM}$ patients compared with those from normal controls as determined by qPCR. HOTAIR expression was obviously increased in MM cell lines compared to that in normal plasma cells. ROC curve analysis showed that the serum level of HOTAIR exhibited a higher diagnostic value for MM. Furthermore, loss-of-functional assays indicated that HOTAIR inhibition suppressed MM cell viability by arresting the cell cycle at the G0/G1 phase as determined by cell viability assay and flow cytometry. An in-depth study revealed that knockdown of HOTAIR led to decreased chemoresistance of DEX in MM cells by regulating the JAK2/STAT3 signaling pathway. Taken together, our findings suggest that upregulation of serum HOTAIR may prove to be a novel biomarker for the diagnosis of MM. HOTAIR promoted MM cell viability and increased chemoresistance of MM to DEX via the JAK2/STAT3
\end{abstract}

Correspondence to: Professor Wei Wang, Department of Hematology, Daqing Oilfield General Hospital, 9 Zhongkang Street, Daqing, Heilongjiang 163001, P.R. China

E-mail:w03weilove@yeah.net

${ }^{*}$ Contributed equally

Key words: HOTAIR, multiple myeloma, biomarker, DEX, JAK2/STAT3 signaling pathway, indicating HOTAIR may also serve as a potential therapeutic target for MM.

\section{Introduction}

Long non-coding RNAs (lncRNAs) are a newly reported type of non-coding RNAs, which are greater than 200 nucleotides in length and are unable to encode proteins (1). IncRNAs are involved in cellular development, genomic imprinting and homeostasis, and numerous studies suggest that dysregulated expression of IncRNAs occurs in various diseases and contributes to tumor biology, including multiple myeloma (MM) (2). For example, Wang et al (3) showed that IncRNA PRAL plays a critical role in the progression of MM, and PRAL could become a novel diagnostic, prognostic and therapeutic candidate for MM patients. Moreover, Zhang et al (4) reported that lncRNA UCA1 promotes MM cell growth by targeting TGF- $\beta$, and overexpression of TGF- $\beta$ partially reverses the effects of UCA1 knockdown. Chen et al (5), identified that lncRNA CCAT1 exerts an oncogenic role in MM by promoting tumor progression, and CCAT1 could serve as a novel diagnostic marker and therapeutic target for MM. However, the associations between others lncRNAs and MM remain unclear, and related reports are lacking.

MM is a type of malignant plasma-cell disorder, which originates from plasma cells of the bone marrow leading to the overproduction of immunoglobulins (6). MM is the second most common hematological malignant tumor, with approximately 80,000 newly diagnosed cases annually worldwide (7). Despite the significant progress in elucidation of the molecular biology of MM and therapeutic options over the past few decades, MM remains incurable, and its 5-year survival rate is approximately $40 \%(8,9)$. Currently, the etiologies and pathogenesis of MM remain poorly understood, and therapies are usually complicated and have poor efficacy. Dexamethasone (DEX) is a key front-line chemotherapeutic agent used for B-cell malignant MM, yet MM cells often become DEX-resistant (10). Therefore, it is urgent to explore the novel functional mechanism of MM and identify new biomarkers to provide effective therapeutic targets for MM. 
In 2007, Rinn et al (11) first identified an IncRNA that regulated gene expression in the trans rather than cis at the HOXC gene locus on chromosome 12, and named the lncRNA as HOX antisense intergenic RNA (HOTAIR). Gupta et al (12), subsequently found that HOTAIR expression is higher in breast cancer, and is associated with tumor metastasis and poor prognosis. Further research showed that HOTAIR is upregulated and plays a significant role in lung cancer, colorectal cancer, hepatocellular carcinoma, nasopharyngeal carcinoma and pancreatic cancer (13). More recently, HOTAIR has been shown to play an oncogenic role in acute myeloid leukemia due to its ability to mediate the migration and invasion of leukemia cells (14). Although HOTAIR plays key roles in different solid cancers and acute myeloid leukemia, little is known concerning its specific function and molecular mechanism in MM.

In the present study, the expression and function of HOTAIR were investigated in MM. Our data showed that the expression levels of HOTAIR were significantly upregulated in serum, bone marrow and primary $\mathrm{CD} 138^{+}$cells from $\mathrm{MM}$ patients compared with those from normal controls, and increased serum HOTAIR may be used as an ideal biomarker for MM detection. Functional assays showed that knockdown of HOTAIR suppressed MM cell viability and arrested the cell cycle in the G0/G1 phase. Furthermore, it was found that HOTAIR increased the chemoresistance of MM to DEX by regulating the JAK2/STAT3 signaling pathway. These data may provide novel theoretical bases for the application of IncRNA HOTAIR in the diagnosis and therapy of MM.

\section{Materials and methods}

MM samples. Peripheral blood and bone marrow samples were collected from $118 \mathrm{MM}$ patients with a mean age of 54.18 years (51 males and 67 females) who were hospitalized at the Department of Hematology, Daqing Oilfield General Hospital from August 2009 to April 2015. Seventy-eight healthy volunteers with a mean age of 56.05 years (36 males and 42 females) were collected as normal controls. All patients were free from other coexisting malignant diseases. Patients with extramedullary myeloma were not enrolled in the present study. No local or systemic treatment had been conducted before operation. For the use of clinical samples for research, the study was approved by the Ethics Committee of Daqing Oilfield General Hospital and performed in accordance with the Declaration of Helsinki (2000). Written informed consent was obtained from all participants. Samples were freshly frozen in liquid nitrogen, and then were stored at $-80^{\circ} \mathrm{C}$ until RNA extraction.

Cell lines and cell culture. MM cell lines (MM.1S, MM.1R, JJN-3 and U266) and normal plasma cells (nPCs) were purchased from the American Type Culture Collection (ATCC, Manassas, VA, USA). All the cells were routinely cultured in RPMI-1640 medium (Thermo Fisher Scientific, Inc.) supplemented with $10 \%$ (vol/vol) FBS (Thermo Fisher Scientific, Inc.) and $1 \%$ penicillin/streptomycin in a $5 \% \mathrm{CO}_{2}$ atmosphere at $37^{\circ} \mathrm{C}$. Primary $\mathrm{CD} 138^{+}$cells were isolated and purified from bone marrow of MM patients and healthy volunteers by using CD138-coated magnetic beads (Miltenyi Biotec,
Paris, France). Primary CD138 ${ }^{+}$cells were freshly frozen in liquid nitrogen, and then were stored at $-80^{\circ} \mathrm{C}$ for further use.

Quantitative PCR ( $q P C R$ ). Total RNA was extracted from cells or samples using TRIzol reagent (Thermo Fisher Scientific, Inc.), according to the manufacturer's instructions. A TaqMan ${ }^{\mathrm{TM}}$ MicroRNA Reverse Transcription kit (cat. no. 4366597; Thermo Fisher Scientific, Inc.) was used to reversely transcribe RNA into cDNA. qPCR was performed with a Mir-X ${ }^{\mathrm{TM}}$ miRNA qRT-PCR TB Green ${ }^{\text {TM }}$ kit (cat. no. 638314; Takara, Beijing, China) on the Applied Biosystems 7500 Sequence Detection system (Thermo Fisher Scientific, Inc.) in triplicate with the following cycling conditions: $95^{\circ} \mathrm{C}$ for $10 \mathrm{~min}$ (initial denaturation), and then 40 cycles of $95^{\circ} \mathrm{C}$ for $10 \mathrm{sec}, 60^{\circ} \mathrm{C}$ for $30 \mathrm{sec}$. The specific primers for HOTAIR, GAPDH, JAK2 and STAT3 were designed and synthesized by GenePharma Co., Ltd. (Shanghai, China). GAPDH was used as an internal control. The primer sequences were as follows: HOTAIR forward, 5'-CCCTCAGGTCCCTAATA-3' and reverse, 5'-CCGCCG TCTGTAACTCT-3'; GAPDH forward, 5'-CCACTCCTCCAC CTTTG-3' and reverse, 5'-ACCACCCTGTTGCTGT-3'; JAK2 forward, 5'-ACCTGGTGAAAGTCCC-3' and reverse, 5'-CCA ATCATACGCATAAA-3'; STAT3 forward, 5'-AGACCCACT CCTTGCC-3' and reverse, 5'-GCTCAGTCCTCGCTTG-3'. Results were normalized to the expression of GAPDH, and the $2^{-\Delta \Delta \mathrm{Cq}}$ method (15) was used for analysis of the quantitative changes in expression of each gene.

Plasmid construction and cell transfection. Lentivirus for short hairpin RNA (shRNA) directed against HOTAIR (Lv-HOTAIR) and control lentiviral vector ( $\mathrm{Lv}$-control) were chemically synthesized from GenePharma Co., Ltd. (Shanghai, China). The sequences of the shRNAs were as follows: 5'-GAA CGGGAGTACAGAGAGA-3' (Lv-HOTAIR) and 5'-GACTGA TTCGATACGCTATGT-3' (Lv-control). Cells were plated in 6-well plates and transfected with Lv-HOTAIR or Lv-control using Lipofectamine 2000 (Thermo Fisher Scientific, Inc.), according to the manufacturer's instructions. Twenty-four $\mathrm{h}$ post-transfection, the transfected cells were selected with $800 \mathrm{mg} / \mathrm{ml} \mathrm{G} 418$ for at least one month. After selection and isolation of stably transfected clones, the clones were analyzed for HOTAIR expression using qPCR assay.

Flow cytometric assay. For analysis of cell apoptosis, the stably transfected cells were co-cultured with $50 \mu \mathrm{M}$ DEX (cat. no. D4902; Sigma-Aldrich; Merck KGaA) for 48 h. Then, the cells were harvested and resuspended in fixation fluid. Propidium iodide (PI) $(2 \mu \mathrm{l})$ and Annexin V-FITC (5 $\mu \mathrm{l})$ were added to the cell suspension for $30 \mathrm{~min}$ at $37^{\circ} \mathrm{C}$. The cell suspension was analyzed using FACSCalibur flow cytometry (BD Biosciences, USA). The percentage of early apoptotic cells was counted and compared for the cells receiving different treatments. For analysis of cell cycle distribution, at $48 \mathrm{~h}$ post-transfection, the cells were harvested via trypsinization, washed with cold PBS and fixed in $70 \%$ ice cold ethanol overnight, followed by staining with $50 \mathrm{mg} / \mathrm{ml}$ PI for $30 \mathrm{~min}$. Finally, the samples were detected with FACSCalibur flow cytometry.

Cell viability assay. Cell viability was measured using a Cell Counting Kit-8 (CCK-8 kit) (Dojindo, USA), according to the 
manufacturer's instructions. Cells were seeded in a 96-well plate at $3 \times 10^{3}$ cells/well and incubated in a $5 \% \mathrm{CO}_{2}$ and $37^{\circ} \mathrm{C}$ humidified incubator with $100 \mu \mathrm{l}$ final volume. After incubation with $50 \mu \mathrm{M}$ DEX for 24, 48, 72 and $96 \mathrm{~h}, 10 \mu \mathrm{l} \mathrm{CCK}-8(5 \mathrm{mg} / \mathrm{ml})$ was added to each well, and the cells were incubated at $37^{\circ} \mathrm{C}$ for $4 \mathrm{~h}$. The absorbance at $450 \mathrm{~nm}$ in each well was detected using a micro-plate analyzer (Bio-Tek Instruments, USA).

Western blot analysis. Cells were lysed with RIPA buffer (Thermo Fisher Scientific, Inc.) according to the manufacturer's instructions, and the concentration of proteins was quantified using a BCA Protein Assay Kit (Beyotime, China). Equal amounts $(30 \mu \mathrm{g})$ of proteins were separated by $10 \%$ SDS-PAGE and electrophoretically transferred onto PVDF membranes (EMD Millipore). After being blocked with $5 \%$ non-fat milk at $37^{\circ} \mathrm{C}$ for $2 \mathrm{~h}$, the membranes were incubated with specific primary antibodies against JAK2 (cat. no. ab108596; 1:1,000), phospho-JAK2 (p-JAK2; cat. no. ab32101; 1:500), STAT3 (cat. no. ab68153; 1:500) and phospho-STAT3 (p-STAT3; cat. no. ab32143; 1:1,000) overnight at $4^{\circ} \mathrm{C}$. GAPDH was used as an internal loading control. All primary antibodies were purchased from Abcam. Finally, the membranes were washed three times with TBST buffer and incubated with HRP-conjugated goat anti-rabbit immunoglobulin G (cat. no. AS014; ABclonal Technology) for $2 \mathrm{~h}$ at $37^{\circ} \mathrm{C}$. The bands were detected using an ECL detection system (Pierce Biotechnology) and analyzed using the Image-Pro plus software (version 6.0; Media Cybernetics Inc.).

Statistical analysis. All results from three independent experiments are expressed as mean \pm SD and were analyzed using Statistical Product and Service Solutions (SPSS) 17.0 (SPSS, Inc., Chicago, IL, USA). Differences among the groups were estimated by Student's t-test or one-way ANOVA followed by Student-Newman-Keuls test. Receiver operating characteristic (ROC) curves were plotted, and the area under the ROC curve (AUC) was calculated to assess the specificity and sensitivity of predicting $\mathrm{MM}$ patients and normal controls. $\mathrm{P}<0.05$ was considered to indicate a statistically significant difference.

\section{Results}

HOTAIR is upregulated in MM samples and cell lines. To explore whether HOTAIR is detectable and altered in MM, the expression of HOTAIR was examined by qPCR in our collection of peripheral blood from $118 \mathrm{MM}$ patients and 78 healthy volunteers. As presented in Fig. 1A, elevated expression of HOTAIR was observed in the serum of MM patients compared with that noted in the normal controls $(\mathrm{P}<0.05)$. We then determined HOTAIR expression in bone marrow samples, and the results revealed that HOTAIR expression in MM patients was significantly higher than that in normal controls $(\mathrm{P}<0.05$; Fig. 1B). To further test the hypothesis that serum HOTAIR was primarily released from tumor cells, the expression level of HOTAIR in primary CD138 ${ }^{+}$cells was also detected. The results showed that HOTAIR expression was significantly upregulated in the primary CD138+ cells from MM patients compared to those from the normal controls $(\mathrm{P}<0.05$; Fig. 1C). Finally, HOTAIR expression was investigated in MM cell lines including MM.1S, MM.1R, JJN-3 and U266, and normal plasma cells (nPCs). The results revealed that the HOTAIR level was considerably increased in the four MM cell lines compared to this level in the nPCs cells ( $\mathrm{P}<0.05$; Fig. 1D). According to the relatively high levels of HOTAIR in MM.1S, U266 and MM.1R cells, and the fact that these cell lines are suitable models for MM as described previously $(16,17)$, three cell lines were selected for further study in vitro.

HOTAIR exhibits a higher diagnostic value for MM. To investigate the characteristic of HOTAIR as a potential diagnostic biomarker for MM patients, ROC curve was performed on data from clinical samples. As presented in Fig. 2, representation of the data showed the AUC of HOTAIR was 0.798 (95\% CI, $0.732-0.865)$, and the sensitivity and specificity were 70.12 and $79.94 \%$, respectively.

Knockdown of HOTAIR suppresses MM cell viability by altering the cell cycle. To further investigate the biological roles of HOTAIR in MM cells in vitro, stable inhibition of HOTAIR expression was established in MM.1S and U266 cell lines using Lv-HOTAIR. As confirmed by qPCR analysis, HOTAIR expression in MM.1S and U266 cells was significantly downregulated by Lv-HOTAIR compared with the expression in the Lv-control-treated and untransfected cells $(\mathrm{P}<0.01$; Fig. 3A). These data indicated that cell transfection was successful, and Lv-HOTAIR is a suitable target to knock down HOTAIR in MM.1S and U266 cells. Then, a CCK-8 assay was performed to detect the role of HOTAIR in the cell viability of MM cells. The results showed that knockdown of HOTAIR inhibited cell viability of the MM.1S and U266 cell lines compared to the cell viability of the Lv-control groups $(\mathrm{P}<0.05$; Fig. 3B and $\mathrm{C})$. To determine whether the function of HOTAIR in regards to cell viability was caused by alteration of the cell cycle, flow cytometry was performed. Cell cycle analysis showed that knockdown of HOTAIR induced MM cell cycle arrest at the G0/G1 phase ( $\mathrm{P}<0.05$; Fig. 3D).

Knockdown of HOTAIR decreases MM chemoresistance to DEX by regulating the JAK2/STAT3 signaling pathway. To investigate whether HOTAIR plays a role in the DEX resistance of MM cells, CCK-8 assay and flow cytometric analysis were carried out to investigate the influence of HOTAIR on the viability and apoptosis of MM cell lines to $50 \mu \mathrm{M}$ DEX. As shown in Fig. 4A and B, knockdown of HOTAIR significantly decreased the chemoresistance of MM.1R cells to $50 \mu \mathrm{M}$ DEX by reducing cell viability at 24, 48, 72 and $96 \mathrm{~h}$, and inducing cell apoptosis at $48 \mathrm{~h}(\mathrm{P}<0.05)$. To confirm whether the effect of HOTAIR on DEX chemoresistance was mediated by the JAK2/STAT3 pathway, qPCR and western blot analysis were performed. The results showed that the mRNA levels of JAK2 and STAT3 in the MM.1R cells demonstrated no significant difference between the Lv-HOTAIR and Lv-control groups (Fig. 5A). Compared with the Lv-control groups, as determined by western blot analysis, expression levels of p-JAK2 and p-STAT3 levels were significantly downregulated in MM.1R cells by HOTAIR inhibition; in contrast, no obvious total protein expression changes in JAK2 and STAT3 were observed between cells receiving different treatments $(\mathrm{P}<0.05$; Fig. $5 \mathrm{~B})$. Taken together, these data suggest that JAK2/STAT3 signaling mediated the effect of HOTAIR on MM chemoresistance to DEX. 
A

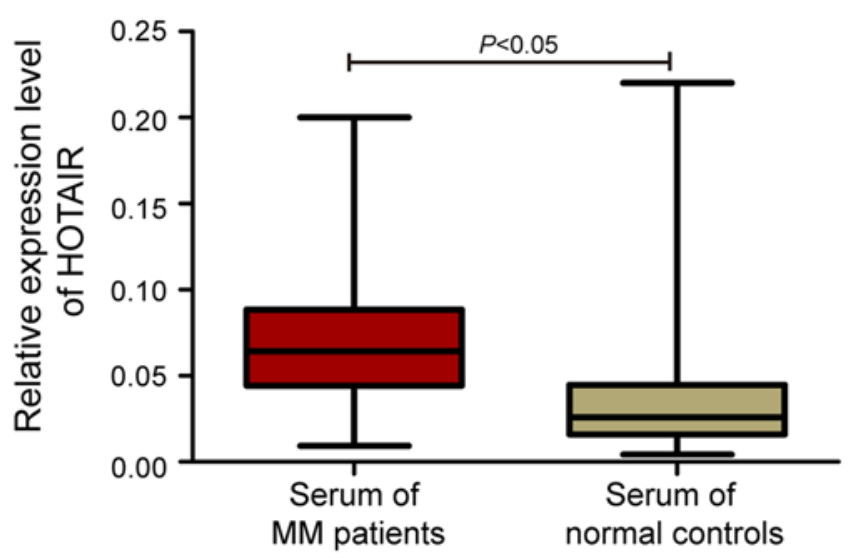

C

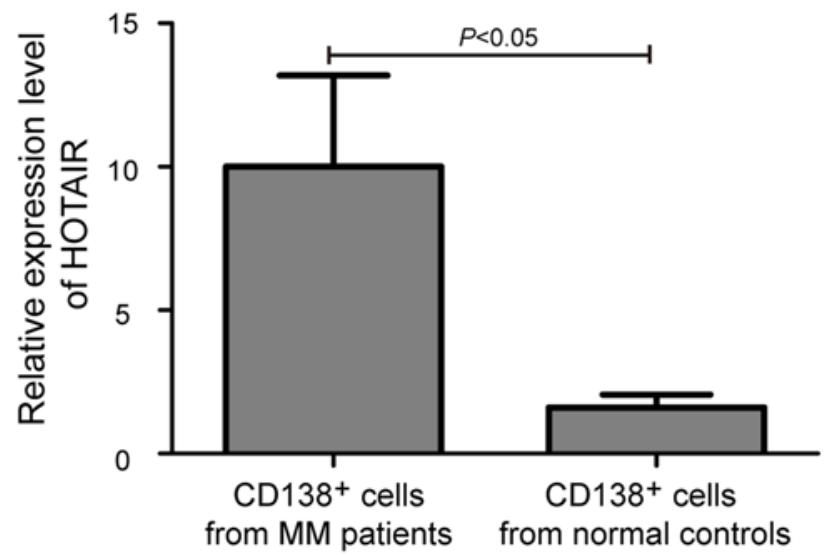

B

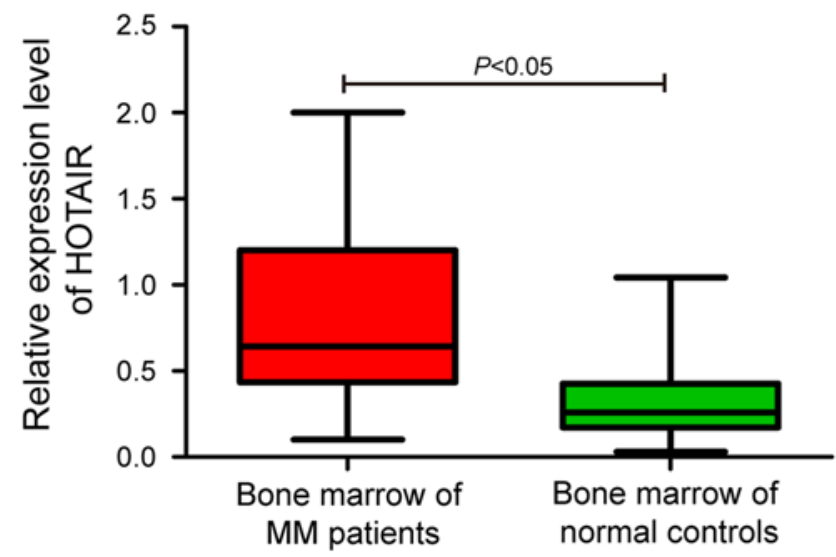

D

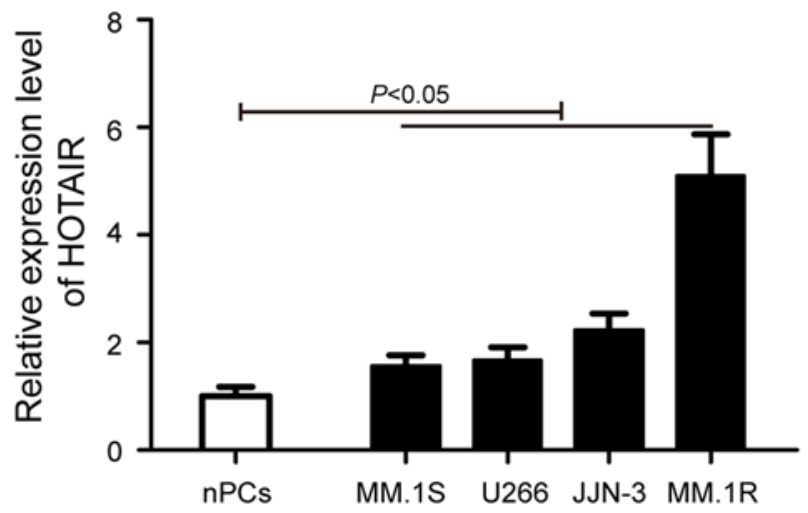

Figure 1. lncRNA HOTAIR is upregulated in MM samples and cell lines. (A) Relative expression of HOTAIR in serum from 118 MM patients and 78 healthy volunteers was detected by quantitative PCR (qPCR). (B) Relative level of HOTAIR in MM patient bone marrow was higher than that in normal control bone marrow. (C) qPCR analysis of the expression level of HOTAIR in primary CD138 ${ }^{+}$cells from MM patients and normal controls. (D) Expression of HOTAIR in four MM cell lines (MM.1S, MM.1R, JJN-3 and U266) and normal plasma cells (nPCs). Values are expressed as the mean \pm standard deviation of three independent experiments. MM, multiple myeloma; lncRNA, long non-coding RNA; HOTAIR, HOX antisense intergenic RNA.

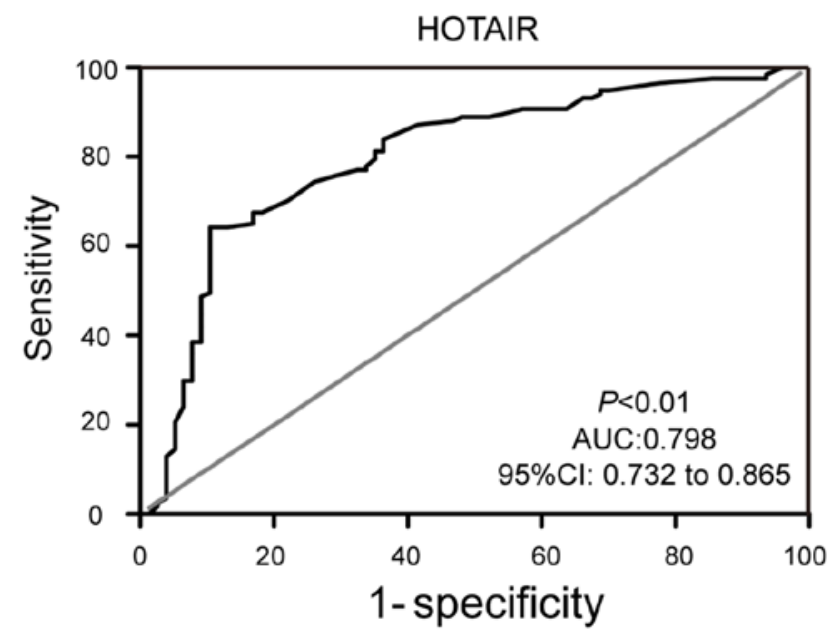

Figure 2. The receiver operating characteristic (ROC) curve of lncRNA HOTAIR expression level in serum for MM diagnosis. The area under the curve (AUC) of HOTAIR was 0.798 (95\% CI: 0.732 to 0.865$)$; the sensitivity and specificity were 70.12 and $79.94 \%$, respectively. Values are expressed as the mean \pm standard deviation of three independent experiments. MM, multiple myeloma; lncRNA, long non-coding RNA; HOTAIR, HOX antisense intergenic RNA.

\section{Discussion}

Multiple myeloma (MM) is a hematological malignancy characterized by abnormal growth of monoclonal plasma cells in the bone marrow leading to various end-organ damage including hypercalcemia, anemia, osteolytic bone disease and renal insufficiency (18). During the past few years, great progress has been achieved in understanding the initiation and tumorigenesis of MM; however, the clinical prognosis of patients with MM still remains highly heterogeneous $(8,9)$. Traditional laboratory biomarkers including $\beta 2 \mathrm{M}, \mathrm{IgG}$ and serum albumin have been used as an objective staging system for MM $(19,20)$. However, these biomarkers have demonstrated limited ability for diagnostic risk stratification. Due to the rapid development of basic medical research, an increasing number of studies have shown that abnormal expression of lncRNAs is detectable in the plasma of cancer patients and therefore may be utilized as a tool for cancer diagnosis $(21,22)$. For example, Shen et al (23) reported that serum lncRNA PCAT-1 is expressed at a higher level in MM patients than that in healthy controls, and may serve 

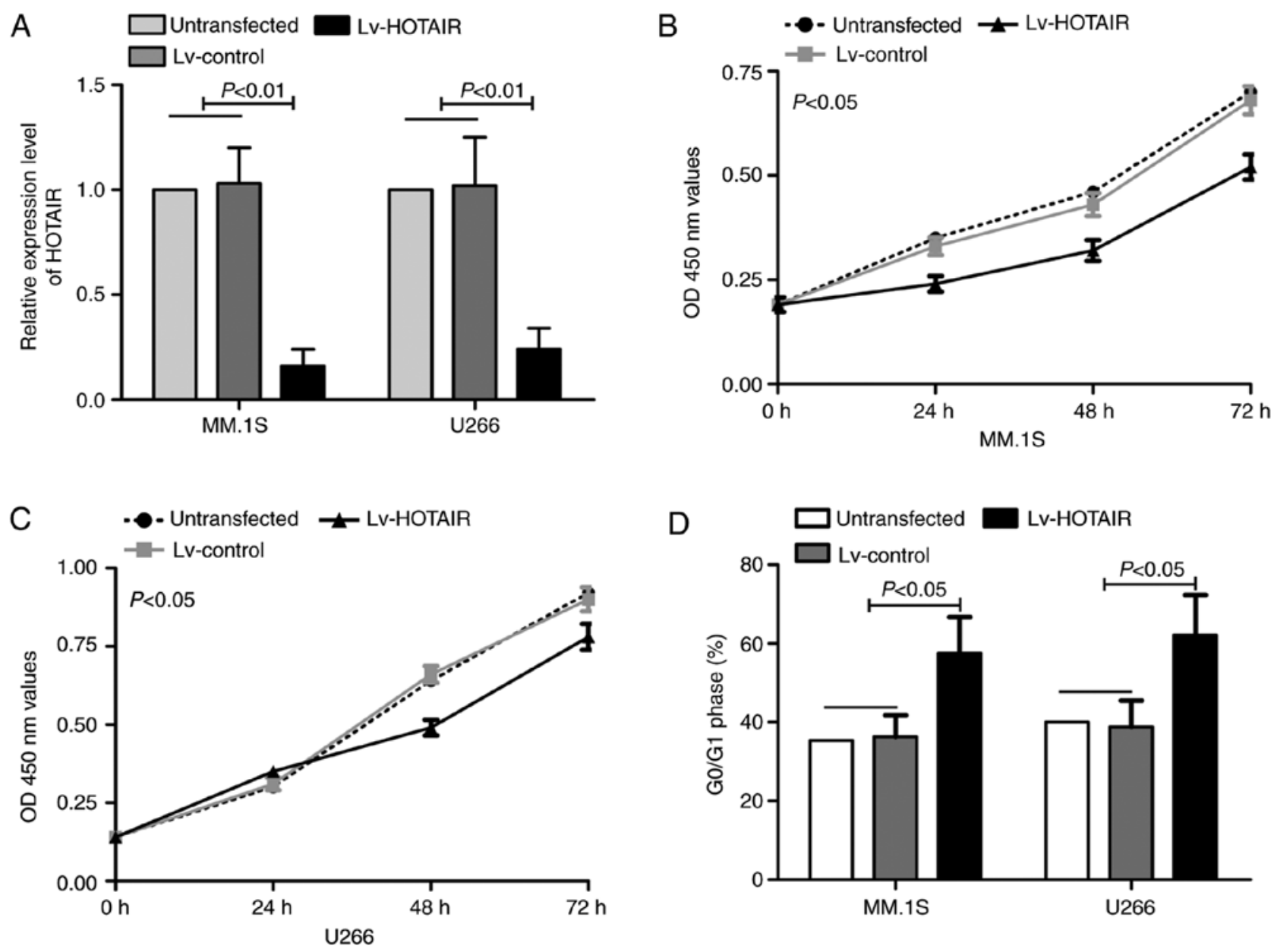

Figure 3. Knockdown of lncRNA HOTAIR suppresses MM cell viability. (A) Relative expression of HOTAIR in MM.1S and U266 cells transfected with Lv-HOTAIR or Lv-control. (B and C) The effect of HOTAIR on MM cell viability was determined by a CCK-8 assay. Knockdown of HOTAIR (Lv-HOTAIR) inhibited cellular viability of MM.1S (B) and U266 cells (C) when compared to the Lv-control groups. (D) The effect of HOTAIR on cell cycle distribution was detected by flow cytometric assay. HOTAIR knockdown induced MM cell cycle arrest at the G0/G1 phase. Values are expressed as the mean \pm standard deviation of three independent experiments. MM, multiple myeloma; IncRNA, long non-coding RNA; HOTAIR, HOX antisense intergenic RNA.
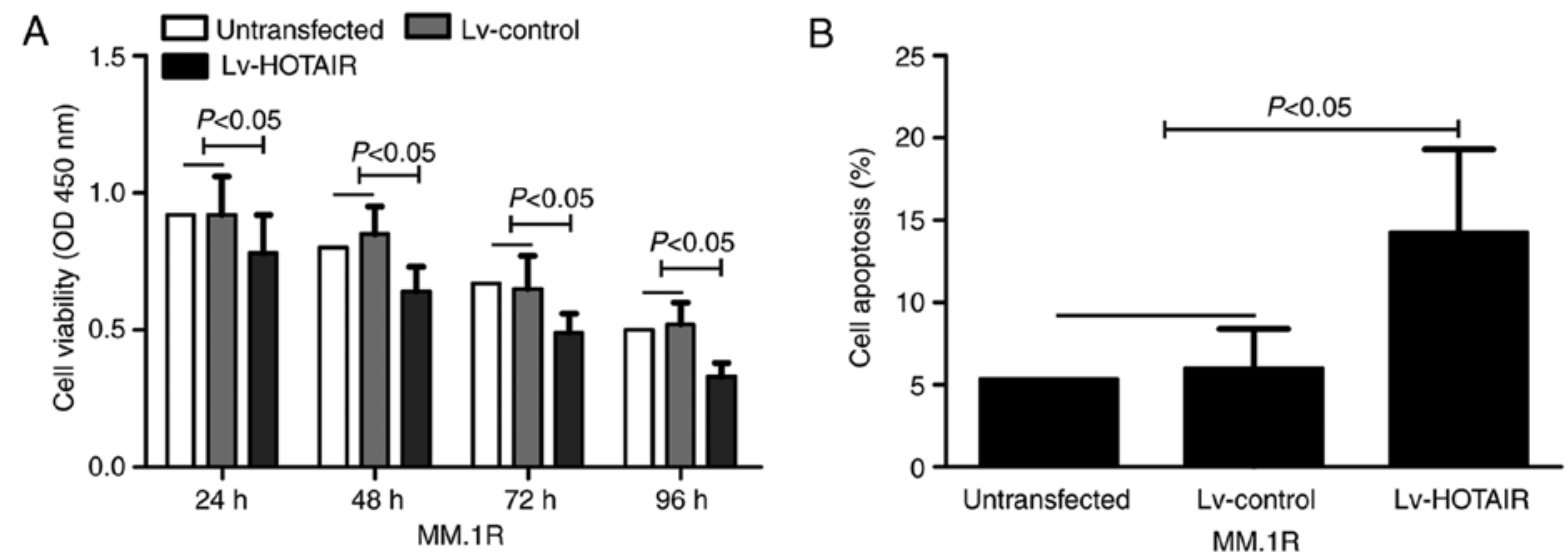

Figure 4. Knockdown of HOTAIR decreases MM chemoresistance to DEX. (A) CCK-8 assay for cell viability of MM cells following treatment of $50 \mu \mathrm{M}$ DEX Knockdown of HOTAIR significantly decreased cell viability of the MM.1R cells at 24, 48, 72 and $98 \mathrm{~h}$ following treatment with DEX. (B) Flow cytometric analysis of cellular apoptosis of MM cells following treatment with $50 \mu \mathrm{M}$ DEX. Knockdown of HOTAIR induced cell apoptosis of MM.1R cells after treatment with DEX at $48 \mathrm{~h}$. Values are expressed as the mean \pm standard deviation of three independent experiments. DEX, dexamethasone; MM, multiple myeloma; lncRNA, long non-coding RNA; HOTAIR, HOX antisense intergenic RNA.

as a valuable biomarker in the auxiliary diagnosis of MM. However, the clinical significance and molecular mechanisms of specific lncRNAs warrant further investigation.
HOTAIR (HOX antisense intergenic RNA), as a spliced and polyadenylated RNA, has six exons with 2.2 -kb nucleotides in length. Accumulating evidence suggests that HOTAIR 
A

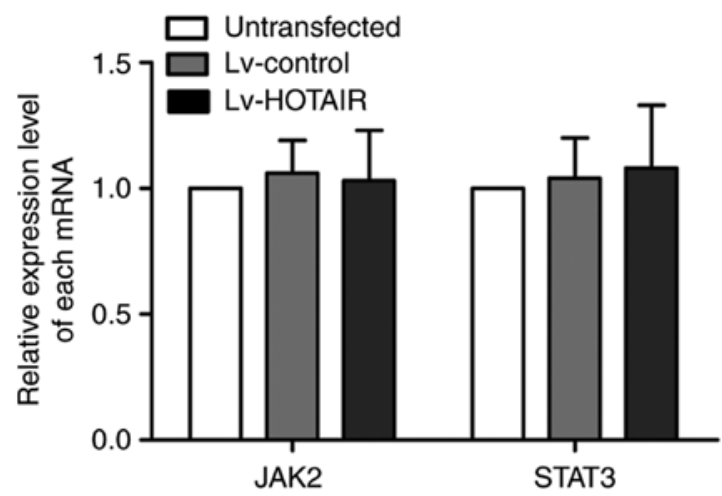

B
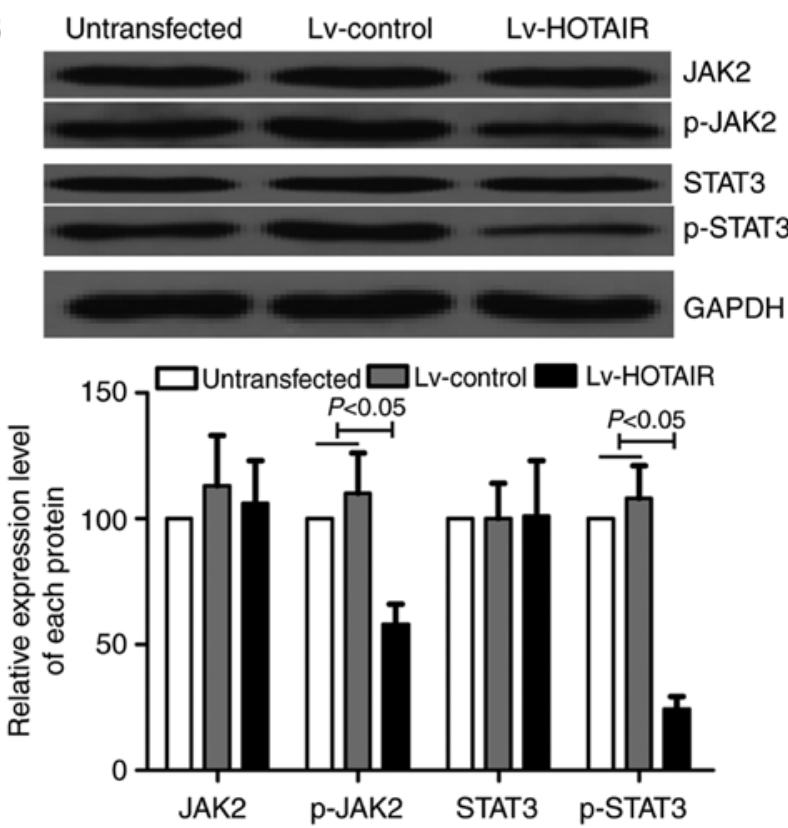

Figure 5. JAK2/STAT3 signaling mediates the effect of IncRNA HOTAIR on MM resistance to DEX. (A) Quantitative PCR analysis of the mRNA expression levels of JAK2 and STAT3 in MM.1R cells in the different transfection groups following treatment with $50 \mu \mathrm{M}$ DEX. (B) Western blot analysis of total protein and phospho(p)-protein expression of JAK2 and STAT3 in MM.1R cells. Knockdown of HOTAIR significantly downregulated the expression levels of p-JAK2 and p-STAT3 in MM.1R cells, whereas no obvious changes in the total protein expression of JAK2 and STAT3 were observed after cells were transfected with Lv-HOTAIR. Values are expressed as the mean \pm standard deviation of three independent experiments. DEX, dexamethasone; MM, multiple myeloma; lncRNA, long non-coding RNA; HOTAIR, HOX antisense intergenic RNA.

plays a critical role in various areas of human cancer, such as growth, survival, invasion, migration, drug resistance and genomic stability (13). Nevertheless, few studies have evaluated the role of serum HOTAIR in the diagnostic value for cancer. A recent study showed that serum HOTAIR could serve as a promising biomarker for diagnosing and monitoring non-small cell lung cancer (24). Elsayed et al (25) concluded that plasma HOTAIR may serve as a potential non-invasive biomarker for the diagnosis of gastric cancer. Tan et al (26) demonstrated that serum HOTAIR could be used as a novel prognostic and diagnostic biomarker for glioblastoma multiforme. In addition, Wang et al (27) reported that serum HOTAIR may serve as a potential biomarker for the diagnosis of esophageal squamous cell carcinoma. However, the diagnostic value and functional role of HOTAIR in MM are still largely unknown. To the best of our knowledge, this is the first study to demonstrate the clinical significance of serum HOTAIR as a diagnostic biomarker for MM. Our results showed a significant upregulation of serum HOTAIR in MM patients compared with that noted in normal controls, and its capability of discriminating between MM patients and healthy volunteers had $70.12 \%$ sensitivity and $79.94 \%$ specificity. Moreover, it was also determined that HOTAIR expression was increased in bone marrow and primary $\mathrm{CD} 138^{+}$cells from MM patients as well as in MM cell lines. Therefore, these data indicated that upregulation of serum HOTAIR may prove to be a novel biomarker for MM diagnosis.

To further investigate the biological role of HOTAIR in MM cells, CCK-8 assay and flow cytometric analysis were carried out to investigate the influences of HOTAIR on the viability and apoptosis of MM cell lines. Function assays showed that silencing of HOTAIR significantly suppressed MM cell viability by arresting the cell cycle in the G0/G1 phase. Dexamethasone (DEX) is one of the most widely used glucocorticoids, which was introduced as a treatment regimen for MM in the 1970s, and its efficacy was observed in up to $70 \%$ of MM patients in clinical trials (28). Recent studies have found that MM cells are resistant to DEX, and some therapeutic strategies for overcoming DEX resistance have been reported (10). For example, miR-221-222 plays a role in DEX-induced drug resistance in MM, and treatment with anti-miR-221-222 was found to partially restore the DEX sensitivity of MM.1R cells (29). NEAT1 inhibition was found to decrease DEX resistance in MM by targeting the miR-193a/MCL1 pathway (16). Importantly, our results showed that knockdown of HOTAIR significantly decreased the chemoresistance of MM.1R cells to DEX by reducing cell viability and inducing cell apoptosis. These data indicate that inhibition of HOTAIR suppresses MM cell viability and decreases DEX resistance.

Signal transducer and activator of transcription 3 (STAT3) is an oncogenic transcription factor and is widely expressed in a broad range of cancers including MM, and plays an important role in regulating cellular activities (30). STAT3 was found to be highly expressed in more than $63 \%$ of $\mathrm{CD} 138^{+}$bone marrow cells of MM patients and is associated with chemoresistance to DEX therapy (31). Currently, the JAK2/STAT3 signaling pathway has been proposed as a promising target for MM therapy (32). To confirm whether the effect of HOTAIR on DEX chemoresistance was mediated by the JAK2/STAT3 pathway, qPCR and western blot analysis were conducted. The data showed that inhibition of HOTAIR significantly downregulated the expression levels of p-JAK2 and p-STAT3 in MM.1R cells, whereas no obvious changes in the mRNA and total protein expression of JAK2 and STAT3 were observed after cells were transfected with Lv-HOTAIR. These important findings suggest that JAK2/STAT3 signaling mediates the effects of HOTAIR in regards to the chemoresistance of MM to DEX.

In summary, our results suggest that serum HOTAIR is a novel biomarker for the diagnosis of MM, and knockdown of HOTAIR was found to inhibit MM cell viability by arresting the cell cycle in the G0/G1 phase. In addition, we conclude that HOTAIR promotes MM chemoresistance to DEX via the JAK2/STAT3 signaling pathway, indicating that HOTAIR may also serve as a potential therapeutic target for MM. 


\section{Acknowledgements}

Not applicable.

\section{Funding}

No funding was received.

\section{Availability of data and materials}

All data generated or analyzed during the present study are included in this published article.

\section{Authors' contributions}

WW designed the study. RG, BF and YP performed the experiments. YL and HL performed the statistical analyses. WW and RG prepared the manuscript. All authors reviewed and edited the final manuscript. All authors read and approved the manuscript and agree to be accountable for all aspects of the research in ensuring that the accuracy or integrity of any part of the work are appropriately investigated and resolved.

\section{Ethics approval and consent to participate}

This study was approved by the Ethics Board of Daqing Oilfield General Hospital and was performed in accordance with the Declaration of Helsinki (2000). Written informed consent was obtained from all participants.

\section{Patient consent for publication}

Not applicable.

\section{Competing interests}

The authors declare that they have no competing interests.

\section{References}

1. Spizzo R, Almeida MI, Colombatti A and Calin GA: Long non-coding RNAs and cancer: A new frontier of translational research? Oncogene 31: 4577-4587, 2012.

2. Nobili L, Ronchetti D, Agnelli L, Taiana E, Vinci C and Neri A: Long non-coding RNAs in multiple myeloma. Genes (Basel) 9 : pii: E69, 2018

3. Wang H, Wang J, Liang CF and Zhou T: Expression of long non-coding RNA PRAL as a potential biomarker for diagnosis in non-small-cell lung cancer patients is associated with the inhibition of cell proliferation and metastasis. Clin Lab 64: 1341-1348, 2018.

4. Zhang ZS, Wang J, Zhu BQ and Ge L: Long noncoding RNA UCA1 promotes multiple myeloma cell growth by targeting TGF- $\beta$. Eur Rev Med Pharmacol Sci 22: 1374-1379, 2018.

5. Chen $\mathrm{L}$, Hu N, Wang $\mathrm{C}$, Zhao $\mathrm{H}$ and $\mathrm{Gu} \mathrm{Y}$ : Long non-coding RNA CCAT1 promotes multiple myeloma progression by acting as a molecular sponge of miR-181a-5p to modulate HOXA1 expression. Cell Cycle 17: 319-329, 2018.

6. Spitzer TR, Sachs DH and Cosimi B: Multiple myeloma. N Engl J Med 364: 2364, 2011.

7. Jemal A, Bray F, Center MM, Ferlay J, Ward E and Forman D: Global cancer statistics. CA Cancer J Clin 61: 69-90, 2011.

8. Kuehl WM and Bergsagel PL: Molecular pathogenesis of multiple myeloma and its premalignant precursor. J Clin Invest 122: 3456-3463, 2012.

9. Anderson KC: Progress and paradigms in multiple myeloma. Clin Cancer Res 22: 5419-5427, 2016.
10. Robak P, Drozdz I, Szemraj J and Robak T: Drug resistance in multiple myeloma. Cancer Treat Rev 70: 199-208, 2018.

11. Rinn JL, Kertesz M, Wang JK, Squazzo SL, Xu X, Brugmann SA, Goodnough LH, Helms JA, Farnham PJ, Segal E and Chang HY: Functional demarcation of active and silent chromatin domains in human HOX loci by noncoding RNAs. Cell 129: 1311-1323, 2007.

12. Gupta RA, Shah N, Wang KC, Kim J, Horlings HM, Wong DJ, Tsai MC, Hung T, Argani P, Rinn JL, et al: Long non-coding RNA HOTAIR reprograms chromatin state to promote cancer metastasis. Nature 464: 1071-1076, 2010.

13. Tang Q and Hann SS: HOTAIR: An oncogenic long non-coding RNA in human cancer. Cell Physiol Biochem 47: 893-913, 2018.

14. Xing CY, Hu XQ, Xie FY, Yu ZJ, Li HY, Bin-Zhou, Wu JB, Tang LY and Gao SM: Long non-coding RNA HOTAIR modulates c-KIT expression through sponging miR-193a in acute myeloid leukemia. FEBS Lett 589: 1981-1987, 2015.

15. Livak KJ and Schmittgen TD: Analysis of relative gene expression data using real-time quantitative PCR and the 2(-Delta Delta C(T)) method. Methods 25: 402-408, 2001.

16. $\mathrm{Wu} \mathrm{Y}$ and Wang $\mathrm{H}$ : LncRNA NEAT1 promotes dexamethasone resistance in multiple myeloma by targeting miR-193a/MCL1 pathway. J Biochem Mol Toxicol 32: Jan, 2018 doi: $10.1002 / j b t .22008$.

17. Li QY, Chen L, Hu N and Zhao H: Long non-coding RNA FEZF1-AS1 promotes cell growth in multiple myeloma via miR-610/Akt3 axis. Biomed Pharmacother 103: 1727-1732, 2018.

18. Kumar SK, Rajkumar V, Kyle RA, van Duin M, Sonneveld P, Mateos MV, Gay F and Anderson KC: Multiple myeloma. Nat Rev Dis Primers 3: 17046, 2017.

19. Moreaux J, Hose D, Reme T, Jourdan E, Hundemer M, Legouffe E, Moine P, Bourin P, Moos M, Corre J, et al: CD200 is a new prognostic factor in multiple myeloma. Blood 108: 4194-4197, 2006

20. Perosa F, Minoia C, Favoino E, Prete M and Dammacco F: Staging multiple myeloma patients with active disease using serum levels of beta2m-free HLA class I heavy chain together with IgM or platelet count. Blood Cells Mol Dis 42: 71-76, 2009.

21. Bhan A, Soleimani M and Mandal SS: Long noncoding RNA and cancer: A new paradigm. Cancer Res 77: 3965-3981, 2017.

22. Bolha L, Ravnik-Glavač M and Glavač D: Long noncoding RNAs as biomarkers in cancer. Dis Markers 2017: 7243968, 2017.

23. Shen X, Zhang Y, Wu X, Guo Y, Shi W, Qi J, Cong H, Wang X, $\mathrm{Wu} \mathrm{X}$ and Ju S: Upregulated lncRNA-PCAT1 is closely related to clinical diagnosis of multiple myeloma as a predictive biomarker in serum. Cancer Biomark 18: 257-263, 2017.

24. Li N, Wang Y, Liu X, Luo P, Jing W, Zhu M and Tu J: Identification of circulating long noncoding RNA HOTAIR as a novel biomarker for diagnosis and monitoring of non-small cell lung cancer. Technol Cancer Res Treat: Jan 1, 2017 doi: $10.1177 / 1533034617723754$ (Epub ahead of print).

25. Elsayed ET, Salem PE, Darwish AM and Fayed HM: Plasma long non-coding RNA HOTAIR as a potential biomarker for gastric cancer. Int J Biol Markers: Apr 1, 2018 doi: 10.1177/1724600818760244 (Epub ahead of print).

26. Tan SK, Pastori C, Penas C, Komotar RJ, Ivan ME, Wahlestedt C and Ayad NG: Serum long noncoding RNA HOTAIR as a novel diagnostic and prognostic biomarker in glioblastoma multiforme. Mol Cancer 17: 74, 2018.

27. Wang W, He X, Zheng Z, Ma X, Hu X, Wu D and Wang M: Serum HOTAIR as a novel diagnostic biomarker for esophageal squamous cell carcinoma. Mol Cancer 16: 75, 2017.

28. Das M: Lenalidomide plus dexamethasone in multiple myeloma. Lancet Oncol 19: e12, 2018.

29. Zhao JJ, Chu ZB, Hu Y, Lin J, Wang Z, Jiang M, Chen M, Wang X, Kang Y,Zhou Y,etal:Targeting themiR-221-222/PUMA/BAK/BAX pathway abrogates dexamethasone resistance in multiple myeloma. Cancer Res 75: 4384-4397, 2015.

30. Arora L, Kumar AP, Arfuso F, Chng WJ and Sethi G: The role of signal transducer and activator of transcription 3 (STAT3) and its targeted inhibition in hematological malignancies. Cancers (Basel) 10: pii: E327, 2018.

31. Liu T, Fei Z, Gangavarapu KJ, Agbenowu S, Bhushan A, Lai JC, Daniels CK and Cao S: Interleukin-6 and JAK2/STAT3 signaling mediate the reversion of dexamethasone resistance after dexamethasone withdrawal in 7TD1 multiple myeloma cells. Leuk Res 37: 1322-1328, 2013.

32. Zhu S, Wang Z, Li Z, Peng H, Luo Y, Deng M, Li R, Dai C, Xu Y, Liu $S$ and Zhang G: Icaritin suppresses multiple myeloma, by inhibiting IL-6/JAK2/STAT3. Oncotarget 6: 10460-10472, 2015. 